\title{
Procedimiento para el perfeccionamiento de la contratación en redes cubanas de frutales y granos
}

\author{
Seida Barrera-Rodríguez ${ }^{*}$ \\ Yeney Acea-Valdés ${ }^{* *}$
}

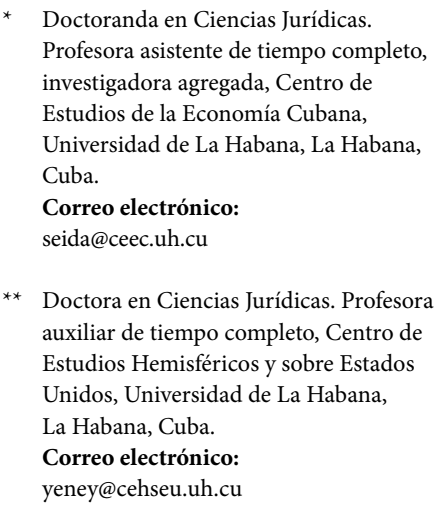

* Doctora en Ciencias Jurídicas. Profesora auxiliar de tiempo completo, Centro de Estudios Hemisféricos y sobre Estados Unidos, Universidad de La Habana,

La Habana, Cuba.

Correo electrónico:

yeney@cehseu.uh.cu

Recibido: 5 de septiembre del 2015

Aprobado: 25 de octubre del 2015

Cómo citar este artículo: Seida BarreraRodríguez, Yeney Acea-Valdés. Procedimiento para el perfeccionamiento de la contratación en redes cubanas de frutales y granos.

DIXI 26. Abril 2016. Pág. 95. doi: http://dx. doi org/10.16925/di.v18i23.1293

\begin{abstract}
Resumen
Introducción: este artículo aborda, desde una perspectiva histórica, los diferentes factores que han incidido en el proceso de contratación de dos redes agrarias cubanas, las cuales abarcan dos provincias del país. Por esta razón, el objetivo principal se centró en proponer un procedimiento para perfeccionarlo. Así mismo, se hace una valoración de las dificultades que han obstaculizado la implementación de las mencionadas redes, tomando como base las principales políticas que rigen el sector. Metodología: fue aplicado el método histórico y el de revisión y análisis de documentos, así como la técnica de entrevista grupal a los 93 asistentes a los cursos impartidos por las autoras en las provincias de Sancti Spíritus y Santiago de Cuba, entre diciembre de 2012 y enero de 2013. Resultado: el presente procedimiento para el perfeccionamiento de la contratación en redes cubanas de frutales y granos. Conclusiones: las herramientas que se le ofrecen al sector jurídico como parte del procedimiento hacen que el contrato agrario refleje en mayor medida la realidad de los actores involucrados y sus necesidades.
\end{abstract}

Palabras clave: contrato, Cuba, red, agricultura. 


\title{
Procedure for Perfecting Procurement in Cuban Fruit and Grain Networks
}

\begin{abstract}
Introduction: this article addresses, from a historical perspective, the different factors that have affected the procurement process in two Cuban agricultural networks comprising two provinces of the country. For this reason, the main objective focused on proposing a procedure for perfecting it. Moreover, it assesses the difficulties that have hindered the implementation of the said networks based on the main policies governing the sector. Methodology: the historical, review and analysis methods as well as the group interview technique were applied to 93 attendees of the courses offered by the authors in the provinces of Sancti Spíritus and Santiago de Cuba, between December 2012 and January 2013. Results: this procedure for perfecting procurement in Cuban fruit and grain networks. Conclusions: the tools offered to the legal sector as part of the procedure cause the agricultural contract to further reflect the reality of stakeholders involved and their needs.
\end{abstract}

Keywords: contract, Cuba, network, agriculture.

\section{Procedimento para o aperfeiçoamento da contratação em redes cubanas de frutas e grãos}

\section{Resumo}

Introdução: este artigo aborda, a partir de uma perspectiva histórica, os diferentes fatores que incidiram no processo de contratação de duas redes agrárias cubanas, as quais abarcam duas províncias do país. Por essa razão, o objetivo principal centrou-se em propor um procedimento para aperfeiçoá-lo. Desse mesmo modo, realiza-se uma avaliação das dificuldades que impediram a implementação das mencionadas redes tomando como base as principais políticas que regem o setor. Metodologia: foi aplicado o método histórico e o de revisão e análise de documentos, assim como a técnica de entrevista grupal aos 93 participantes dos cursos ministrados pelas autoras nas províncias de Sancti Spíritus e Santiago de Cuba, entre dezembro de 2012 e janeiro de 2013. Resultado: o presente procedimento para o aperfeiçoamento da contratação em redes cubanas de frutas e grãos. Conclusões: as ferramentas que são oferecidas ao setor jurídico como parte do procedimento fazem com que o contrato agrário reflita em maior medida a realidade dos atores envolvidos e suas necessidades.

Palavras-chave: contrato, Cuba, rede, agricultura. 


\section{INTRODUCCIÓN}

En el plano internacional, la alimentación ha pasado a convertirse en una cuestión de seguridad estratégica. En el caso de los países en vías de desarrollo como Cuba, donde la explotación de sus recursos naturales se escapó durante siglos hacia el exterior hasta el triunfo de la Revolución en 1959, la producción agraria constituye un pilar fundamental en la lucha por la independencia alimentaria. El bloqueo norteamericano que aún persiste, el cambio climático y el impacto negativo de la crisis sistémica que está afectando al mundo son solo algunas de las causas que ubican al país en una situación vulnerable y obligan a extraer de la tierra el mayor beneficio posible.

En ese afán de aprovechamiento de la tierra como recurso natural, la comercialización de los productos que de ella se obtienen ocupa un lugar cimero en la red de producción-distribución. Esto se debe a que permite que el esfuerzo realizado por los productores durante meses, incluso años (según el ciclo productivo de cada actividad), se materialice en términos sociales - ya sea por medio de la entrega de productos para el consumo de la población, la industria y las entidades acopiadoras, o bien por medio de la percepción de ingresos que recompensen la labor realizada-, los cuales posibilitan a su vez la adquisición del financiamiento para el inicio de un nuevo ciclo productivo.

En Cuba ha prevalecido la presión sobre los productores para la contratación de sus cosechas, mientras que las entidades acopiadoras o distribuidoras de insumos no logran garantizar los suministros necesarios para la ejecución de los acuerdos negociados. Esta situación deviene en un círculo vicioso al impedir el cumplimiento de los compromisos contraídos por los productores, a pesar de sus esfuerzos enfocados en resolver con medios propios y las tecnologías alternativas las limitaciones impuestas. En este sentido, es loable destacar el valor de los conocimientos locales y tradicionales agrícolas, que si bien no gozan de una base científica, constituyen verdaderas alternativas ante los problemas. Se trata de saberes y experiencias que se transmiten entre generaciones o entre campesinos que pertenecen a una localidad determinada, con la impronta que exige cada momento, de manera que no permanecen inalterables.

El presente material formó parte de la capacitación que dictaron las autoras de este artículo a productores, representantes de los gobiernos provinciales, investigadores y empresas que le brindan servicios al sector agrario como parte de un programa conjunto con el Ministerio de la Agricultura, el cual priorizó las redes de frutales y granos, y fue financiado por el Programa de las Naciones Unidas para el Desarrollo (PNUD, por sus siglas en inglés). Dicho proyecto se conoció en su momento con el nombre de Palma (Programa de Apoyo Local a la Modernización Agraria en Cuba), y hoy tiene su continuación en el proyecto denominado Gestión Integral Cooperativa.

El objetivo principal se centró en proponer un procedimiento que permita perfeccionar la comercialización de los productos del agro, como alternativa ante las problemáticas halladas en las redes del sector.

La metodología incluyó la aplicación del método histórico y el de análisis de documentos, así como la técnica de entrevista grupal a los 93 asistentes a los cursos (de los cuales 62 son productores) impartidos por las autoras en las provincias de Sancti Spíritus y Santiago de Cuba, entre diciembre del 2012 y enero del 2013. Los análisis fueron sintetizados y actualizados en enero del 2014.

\section{A. Breve ubicación del contexto histórico}

En las relaciones de Cuba con el exterior, impacta hoy de manera negativa la crisis sistémica que afecta al mundo, lo cual ha ocasionado que aumenten los precios de los alimentos e insumos necesarios para ejecutar las actividades agrarias. Las naciones más desarrolladas han optado por tomar el control sobre zonas estratégicas, utilizando la guerra preventiva o la cibernética. Los medios audiovisuales y la publicidad bombardean a la población, induciéndola a un consumo desenfrenado e insostenible económicamente. A dicha situación se suma el bloqueo del Gobierno de Estados Unidos, el cual pone a los negociadores cubanos en una posición muy difícil respecto a los precios, lugares de entrega y requerimientos de calidad (entre otras condiciones), coyuntura que conoce la contraparte extranjera. La violación de normas de Derecho internacional como el Acuerdo General sobre Aranceles de 1944 o el Acuerdo de Marrakech, por el que se constituye la Organización Mundial de Comercio, se ha convertido en un hecho cotidiano.

La situación anterior se traduce en una situación desventajosa, que reduce la disponibilidad de crédito en los contratos internacionales y ocasiona la imposición de condiciones leoninas. Además, la tecnología y, en

1. Véase Rodolfo Dávalos Fernández. ¿Embargo o bloqueo? LA instrumentación de UN CRIMEN CONTra Cuba. Capitán San Luis. (2012). Pág. 180 
general, los resultados de investigación sirven mayoritariamente a los grandes capitales, de manera que casi cualquier producto llega a las tiendas con una fecha límite de uso que cada vez se acorta más: la obsolescencia programada. ${ }^{2}$ Igualmente, la desaparición paulatina del mercado de piezas de repuesto hace que las empresas estatales cubanas se vean forzadas a gastar de manera periódica en la renovación del equipamiento. Todo ello ha ocasionado que la cuestión alimentaria se considere un tema de seguridad nacional para el país, lo que explica la concentración de esfuerzos y financiamiento en las áreas agrarias.

Frente a la tensa situación antes descrita, Latinoamérica se encuentra dando pasos a fin de buscar la integración por medio de la Alianza Bolivariana para las Américas (ALBA), la Comunidad de Estados del Caribe (CELAC) o la Caribbean Community (CARIсом). Cuba ha logrado negociar nuevos plazos de pago de su deuda externa, y ha conseguido nuevas líneas de crédito con naciones como Brasilo Venezuela, ganando con ello credibilidad ante los inversionistas extranjeros.

En el contexto descrito, el Decreto Ley 304 de 2012 (De la contratación económica), se considera uno de los logros de los lineamientos que antecedieron a las transformaciones del modelo económico cubano a partir del $2011,{ }^{3}$ pues logró acabar con décadas de dispersión legislativa, cuya mayoría se remonta a la década de los ochenta. Esto se demuestra en la disposición final segunda, ya que deroga 12 cuerpos jurídicos total o parcialmente, a los que se pueden sumar aquellos que se engloban en la disposición final tercera que, a manera de "saco", deroga las disposiciones de igual o inferior jerarquía que se opongan a la mencionada norma.

Igualmente, con los lineamientos se ha logrado perfeccionar la visión sistémica del acto de legislar, pues a partir de aquí los organismos involucrados en el dictado de una norma jurídica trabajan más estrechamente. Por ejemplo, antes una ley concedía un término para que se dictara el reglamento luego de entrar en vigencia, como sucedió con la Ley 105 de

\footnotetext{
2. Véase Dunia Castillo Galán. Obsolescencia programada. Juventud Rebelde, edición digital. Mayo 15 de 2013; Óscar Arambulo Reyes. ENSAYO DE LA OBSOLESCENCIA PROGRAMADA. Instituto de Postgrado y Educación Continua, Universidad de Guayaquil. (2013); Kathleen Fitzpatrick. Planned obsolescence: PUBLISHING, TECHNOLOGY, AND THE FUTURE OF THE ACADEMY New York University Press. (2011).

3. Véase Partido Comunista de Cuba. Lineamientos de la Polí tica eCONómica y social del Partido y la Revolución. Partido Comunista de Cuba. (2011)
}

2009 de Seguridad Social, que concedió 60 días para ello. Sin embargo, el reglamento se promulgó a los 92 días, lo cual no hubiera tenido mayores consecuencias si no fuera porque algunos órganos a nivel local malinterpretaron que la espera implicaba detener temporalmente los procedimientos presentados, lejos de continuar aplicando la ley anterior. ${ }^{4}$ Ahora se trabaja por temas, los cuales son publicados en la Gaceta Oficial de la República como compendio, buscando la mayor integralidad posible, tal como sucedió con las cooperativas no agrarias. ${ }^{5}$

\section{B. Dificultades actuales con la contratación en redes de frutales y granos}

Los principales incumplimientos planteados por los miembros de las redes de frutales y granos que fueron capacitados, se clasificaron de acuerdo con la posición que ocuparon en el ciclo del capital. Para el que provee, estuvieron en la entrega tardía de los insumos destinados a producir y a la transportación. Para el que vende, las mayores dificultades se hallaron en las entregas planificadas de productos y sus requerimientos de calidad. Nótese cómo los proveedores y vendedores señalaron en todo momento factores externos a su labor individual. Sin embargo, cuando los contratos que supuestamente los amparaban fueron revisados, se advirtió que los elementos mencionados presentaron una redacción deficiente en las condiciones de contratación que debieron haberlos reflejado, y no previeron alternativas a seguir cuando se tipificaron.

Los altos índices de formalismo determinaron que la meta era lograr que los directivos autorizados hicieran la rúbrica de los documentos. El contrato se reservó en muchos casos para ser mostrado en una inspección, en vez de utilizarlo como herramienta cotidiana de consulta y negociación. Cabe señalar que los productores presentes en los cursos impartidos se

\footnotetext{
4. Véase Ley 105 de 2009. Ley de Seguridad Social. Enero 22 de 2009. GOE N. ${ }^{\circ}$ 004; Decreto 283 de 2009. Reglamento de la Ley de Seguridad Social. Abril 24 de 2009. GOE N. ${ }^{\circ} 013$.

5. Véase Decreto Ley 305 de 2012. De las Cooperativas no Agropecuarias. Diciembre 11 de 2012. GOE N. ${ }^{\circ}$ 53; Decreto Ley 306 de 2012. Del régimen especial de seguridad social de los socios de las Cooperativas no Agropecuarias. Diciembre 11 de 2012. GOE N. ${ }^{\circ}$ 53; Decreto 309 de 2012. Reglamento de las Cooperativas no Agropecuarias de primer grado. Diciembre 11 de 2012. GOE N. ${ }^{\circ}$ 53; Resolución 570 de 2012. Para licitar los bienes de un establecimiento estatal para su gestión por las Cooperativas no Agropecuarias. Diciembre 11 de 2012. GOE N. ${ }^{\circ}$ 53; Resolución 427 de 2012. Que estableció indicaciones para el régimen tributario de los cooperativistas no agropecuarios. Diciembre 11 de 2012. GOE N. ${ }^{\circ} 53$.
} 
encuentran entre los mejores del país, y todos tenían una larga experiencia en la contratación con empresas, pequeños agricultores, granjas estatales o cooperativas. No obstante, los defectos en los contratos fueron imputables a las partes involucradas y afectaron a todos por igual, aunque nadie se autocriticara por este particular. Fue realmente difícil hacerles ver la importancia del contrato, cómo podían invocarlo ante un incumplimiento y, sobre todo, convencerlos de abandonar la costumbre de usar proformas, las cuales en ocasiones dejaban fuera cuestiones clave en cuanto a la solicitud de responsabilidad a la contraparte.

Lograda la persuasión, quedaron pendientes las cuestiones materiales para asegurar el uso correcto del contrato. Si resultó trabajoso demostrarles a los miembros de la red lo que podían lograr con un negocio bien protegido, fue aún más difícil convencerlos de que debían invertir en papel, en tinta o en el pago del jurista que los atendía, pues ahora tendrían que acudir con mayor frecuencia a visitarlos, con el fin de darles un mayor seguimiento a los contratos que dejarían de ser meras proformas. Algunos productores y distribuidores plantearon que simplemente no podrían asumir los costos del perfeccionamiento contractual, y por problemas de financiamiento nunca se regresó a las provincias para comprobar in situ los avances o retrocesos de aquellos que decidieron seguir los consejos de las autoras.

Las causas del comportamiento antes descrito coinciden con un informe de la Dirección de Asesoramiento Jurídico del Ministerio de Justicia, en el que se plantea que las bases productivas agrarias son reincidentes en la no contratación de juristas, bien sea porque no destinan presupuesto para ello, piensan que no es necesario o bien utilizan al que labora en la dirección superior. ${ }^{6}$ Además, el desconocimiento o la poca conciencia de la importancia del vínculo contractual provoca que en ocasiones se abuse de los denominados contratos por adhesión, cuando el artículo 17 del Decreto Ley N. ${ }^{\circ} 304$ establece que, por lo general, se utilizan para entidades cuyo alto volumen de clientes les impide establecer procesos de negociación, como es el caso de las sucursales bancarias, de seguros y de servicios básicos.

Según los resultados de una investigación entregada en el 2013 por el Centro de Investigaciones Jurídicas del Ministerio de Justicia de Cuba, los

\footnotetext{
6. Véase Dirección de Asesoramiento Jurídico del Ministerio de Justicia. Situación ACTUAL DE LA ACTIVIDAD DE ASESORAMIENTO JURÍDICO EN LAS ENTIDADES QUE INTEGRAN LA ECONOMÍA NACIONAL. Ministerio de Justicia. (2012).
}

consultores jurídicos plantearon entre las mayores inquietudes relacionadas con el contrato las siguientes:

1. Se imponen cifras de cumplimiento de la persona jurídica que no satisfacen al campesino.

2. No se tiene en cuenta el clima como posible caso fortuito o de fuerza mayor.

3. Existe carencia de un diagnóstico efectivo de las potencialidades de los productores.

4. Se acude tardíamente al asesoramiento jurídico. Olvidan su carácter preventivo. ${ }^{7}$

Por parte de la Dirección Jurídica del Ministerio de la Agricultura, se suma lo siguiente:

5. Imprecisiones en los cronogramas de entrega de productos que obliguen a las partes a entregar y recibir en fecha la cantidad y calidad determinada.

6. Falta de credibilidad de los productores en cuanto a la disponibilidad en tiempo de los insumos y/o servicios productivos que deben brindar las empresas agrarias y/o de suministros agrarios.

7. No se negocian y firman oportunamente los contratos con los proveedores de insumos y/o servicios. ${ }^{8}$

La doble moneda es otra dificultad para la que tampoco se avizora solución a corto plazo, pero más preocupantes son las carencias de autorizaciones para operar en divisa de algunos de los miembros de la red, tales como las cooperativas. Lógicamente, se comprende que debe ser un proceso gradual. Sin embargo, las negociaciones no pueden pararse por este motivo, por lo cual se requieren alternativas temporales y liberación de trabas para este sector. Durante la visita a Santiago de Cuba en enero de 2013, con el ánimo de capacitar a los empresarios que intervendrían en la red, se presentó el caso del representante de una cooperativa de producción agraria que en ocasiones percibe divisa por sus productos y debe recurrir, a su cuenta y riesgo, a la utilización de la cuenta de la entidad que lo atiende, la cual sí puede operar en dicha moneda. La consecuencia ha sido que, en más de una ocasión, ha solicitado la extracción de efectivo y han hallado que la cuenta está vacía o que no se puede acceder por congelamiento ante demandas judiciales,

\footnotetext{
7. Véase Gerardo Machado, et al. El asesoramiento jurídico a LAS COOPERATIVAS AGRARIAS. INFORME FINAL DE INVESTIGACIÓN. Centro de Investigaciones Jurídicas. (2013).

8. Véase Ministerio de la Agricultura. Políticas y procediMIENTOS A SEGUIR PARA LA CONTRATACIÓN DE LA PRODUCCIÓN Agropecuaria y forestal para el aÑo 2014. Ministerio de la Agricultura. (2013)
} 
sin poder exigir nada y afectando sus ingresos, pues nadie cubre los costos en los que incurrió para obtener y vender sus producciones.

Las cooperativas no agrarias podrían constituir un alto en el camino en el propósito de liberar de obstáculos los enlaces, debido a su capacidad para confiar a particulares actividades que el Estado sencillamente no puede asumir, y que son tan necesarias para el éxito como el ciclo de plantas y animales para el cultivo y la crianza. Hablamos del transporte, la construcción y el mantenimiento de equipos, entre otros. No obstante, por el momento solo constituyen experimentos y el mecanismo para su aprobación es muy difícil, pues solo la elevación y evaluación por la Comisión de Implementación de los Lineamientos, y posteriormente por el Consejo de Ministros, puede demorar meses.

A manera de síntesis, se encontraron varias tendencias:

1. Poco o nulo conocimiento de los miembros de la red de frutales y granos sobre el papel del contrato, cuestión que forma parte de un fenómeno de mayor magnitud, que abarca a la ciudadanía en su conjunto en temas jurídicos generales. Este problema ya ha sido detectado por el Centro de Investigaciones Jurídicas, lo cual ha motivado medidas diversas de la Dirección de Divulgación del Ministerio de Justicia, pero aún son evaluadas como insuficientes. ${ }^{9}$

2. Existen limitaciones importantes del Estado a la actividad de los sujetos económicos y mercantiles que están involucrados en la red, para los cuales no se avizoran alternativas o no serán de manera inmediata. Es necesario entonces alertar sobre aquellas cuestiones que pueden no haberse detectado, y evaluar el impacto en todos los órdenes que han ocasionado las medidas en fase de implementación.

\section{Procedimiento para el diagnóstico de la red de frutales y granos en materia contractual}

A partir de los resultados ya explicados se determinó proponer un procedimiento, producto de un diagnóstico realizado a finales del 2013. Las sugerencias que a continuación se exponen fueron elaboradas en conjunto con las partes contratantes, lo cual enriqueció la propuesta. El objetivo fue economizar al máximo el tiempo, a fin de declarar lista la red de frutales y granos, y no detectar problemas que luego necesitarán de un segundo control para verificar su erradicación.

1. El primer paso es identificar a los especialistas que deben acompañar todo el proceso contractual y evaluar su intervención (operador logístico, directivos, tecnólogos, epidemiólogos, etc.). Respecto al asesor jurídico, verificar si todas las entidades involucradas poseen uno propio o contratado, dados los problemas detectados en esta área. Chequear el conocimiento sobre las diferentes formas de pago al aesor (por asunto, iguala $u$ horario) y cuál es el elegido. Hacer recomendaciones sobre la adquisición del servicio en los casos en los que se advierta su carencia.

2. En el caso específico de las redes de frutales y granos, dado el grado de desconocimiento sobre la temática que hay entre sus miembros, resulta imprescindible realizar talleres previos de sensibilización, no con el propósito de capacitar a los productores y directivos, sino para contribuir a crear una conciencia de red.

3. Realización de diagnóstico: revisar si todas las relaciones jurídicas intermiembros se encuentran protegidas mediante contrato formalizado, según los requerimientos de los Decretos Leyes 304 y 310, así como cuáles son las dificultades históricas respecto a su negociación y ejecución. Dado el volumen de este tópico y las ideas que aportan las normas jurídicas antes citadas, resulta poco lo que puede quedar a la imaginación del asesor jurídico, por lo que se convierten de hecho en guías que ahorran tiempo y previenen desacuerdos. Es necesario explorar la voluntad de las partes respecto a varios detalles que a continuación se expondrán, a fin de saber en qué medida fueron tenidos en cuenta y recomendar su análisis en caso de que no se hayan revisado. Se propone la aplicación del siguiente ejemplo de instrumento:
9. Véase Silvia García Méndez, Mercedes de Armas Alonso y Karima Sánchez Rey. Miradas cruzadas: comunicación y cultura jurídica. Anuario del Centro de Investigaciones JURÍDICAS 2009-2010. Centro de Investigaciones Jurídicas. (2010). 
Tabla 1. Guía de diagnóstico del contrato de producción y/o servicios de frutales y granos

\begin{tabular}{|c|c|c|}
\hline N. ${ }^{\circ}$ & Aspecto a revisar & Puntaje \\
\hline 1. & $\begin{array}{l}\text { Correcta acreditación e identificación de las partes, ver si se aportaron copias de la documentación a exhibir o si } \\
\text { se suscribió un certificado como constancia por los asesores jurídicos. }\end{array}$ & 3 \\
\hline 2. & Un mismo consultor, abogado o asesor jurídico no representa a dos o más miembros de la red. & 3 \\
\hline 3. & Tratos preliminares protegidos, con determinación de la responsabilidad precontractual. & 3 \\
\hline 4. & $\begin{array}{l}\text { Existencia de contrato por adhesión o mediante bases permanentes que deben haber sido negociadas libre- } \\
\text { mente. En todo caso, se cuidará de que no existan cláusulas abusivas que eximan de responsabilidad a alguna de } \\
\text { las partes injustificadamente. }\end{array}$ & 3 \\
\hline 5. & $\begin{array}{l}\text { Se expresa la voluntad de las partes respecto al subcontrato y se establece la responsabilidad ante terceros. } \\
\text { Como los contratos iniciales serán solo entre nacionales, no se tendrán en cuenta los términos comunes y } \\
\text { tratados internacionales. }\end{array}$ & 3 \\
\hline 6. & $\begin{array}{l}\text { El objeto y los plazos de cumplimiento están determinados para todos los momentos de ejecución. Se establece } \\
\text { la responsabilidad en caso de incumplimiento en su aceptación. }\end{array}$ & 3 \\
\hline 7. & $\begin{array}{l}\text { Se garantizan las obligaciones con sanción pecuniaria, avales bancarios, fianza u otra de las formas reconocidas } \\
\text { en la ley. }\end{array}$ & 2 \\
\hline 8. & Se establecen reglas para la interpretación o se recurre a los artículos del Decreto Ley 304. & 1 \\
\hline 9. & $\begin{array}{l}\text { Desde el contrato principal se determinan los requerimientos de calidad de los productos o servicios que se } \\
\text { ofrecerán. }\end{array}$ & 3 \\
\hline 10. & $\begin{array}{l}\text { Seguro con clara regulación en póliza sobre quién obtiene la indemnización y qué riesgos cubre. Se comparten } \\
\text { los riesgos en dependencia de la operación. }\end{array}$ & 1 \\
\hline 11. & Se prevén soluciones alternativas ante conflictos. & 1 \\
\hline 12. & Existe voluntad respecto a la exclusividad. & 1 \\
\hline 13. & Están delimitadas las situaciones en las que se recurrirá a los avisos o advertencias. & 2 \\
\hline 14. & $\begin{array}{l}\text { Están definidas las causas de limitación o excepción de responsabilidad (caso fortuito o fuerza mayor por clima, } \\
\text { accidente no imputable al obligado, etc.). }\end{array}$ & 3 \\
\hline 15. & Ante la existencia de litigios, se establece el tribunal o árbitro que conocerá del caso. & 2 \\
\hline 16. & Se refleja el periodo de vigencia del contrato, las causales de extinción, de resolución y prescripción. & 3 \\
\hline 17. & De existir modificaciones, se reflejan tanto en el contrato principal, como en los suplementos. & 3 \\
\hline
\end{tabular}

Fuente. Elaboración propia a partir de los parámetros del Decreto Ley 304

De las verificaciones antes expuestas, se han protegerse para toda la red, más allá del mero condetectado siete hasta ahora, las cuales deberían tacto interpartes.

Tabla 2. Aspectos a verificar para toda la red de frutales y granos

\begin{tabular}{|c|l|c|}
\hline N. & \multicolumn{1}{|c|}{ Aspecto a revisar } & Puntaje \\
\hline $\mathbf{1 .}$ & $\begin{array}{l}\text { Que a partir de la identificación de las partes se pongan todos los contactos de los miembros de la red, así como } \\
\text { de los potenciales candidatos a convertirse en miembros. }\end{array}$ & $\mathbf{1}$ \\
\hline $\mathbf{2 .}$ & Se protegen los encargos futuros en el caso de los suministros. & $\mathbf{1}$ \\
\hline $\mathbf{3 .}$ & Existen estudios sobre los costos antes y después de la aplicación del procedimiento. & $\mathbf{1}$ \\
\hline $\mathbf{4 .}$ & Que se hayan detectado sustitutos con capacidad para cumplir una obligación puntual, en caso de contingencia. & $\mathbf{1}$ \\
\hline $\mathbf{5 .}$ & $\begin{array}{l}\text { Límites de producción, comercialización y distribución consensuados a nivel de red. De esta manera, se evitarán } \\
\text { los desequilibrios ante las diferentes capacidades de los miembros y se prevendrán litigios. }\end{array}$ & $\mathbf{1}$ \\
\hline $\mathbf{6 .}$ & Están delimitadas las situaciones en las que se recurrirá a los avisos o advertencias. & $\mathbf{1}$ \\
\hline $\mathbf{7 .}$ & $\begin{array}{l}\text { Todos los miembros de la red poseen pólizas de seguro, y se regula claramente en el contrato quién obtiene la } \\
\text { indemnización y qué riesgos cubre. Se comparten los riesgos en dependencia de la operación. }\end{array}$ & $\mathbf{1}$ \\
\hline
\end{tabular}


Se propone la siguiente evaluación.

Puntaje durante el diagnóstico. Solo se evaluará hasta el punto 17, ya que se conoce de antemano que ninguno de los contratos contiene el enfoque de red. Suman 40 puntos en total.

+30 a 40 (100\%) puntos: BIEN

+20 a 30 (75\%) puntos: REGULAR

0 a $20(50 \%)$ puntos: MAL

Puntaje después de aplicado el procedimiento. Se evaluará del punto 1 al 16, y se otorgará otra calificación del 17 al 24. Esta última decidirá si el contrato es apto para utilizarse en redes de producción y/o servicios.

+22 a 24 puntos: BIEN

+20 a 22 puntos: REGULAR

18 a 20 puntos: MAL

\section{Pros y contras de las variantes de un contrato de producción $y /$ o servicios de frutales y granos}

En este punto, resulta conveniente decidir si debe crearse un contrato con múltiples partes en el cual estarán representados todos los miembros, o redactar desde cada contrato interparte cláusulas específicamente relacionadas con la red.

En cuanto al contrato multipartes, a favor puede señalarse que es el más integrador desde el punto de vista negocial. Sin embargo, dificulta la particularización de la solicitud de responsabilidad. De otra parte, tanto la negociación como la redacción resultarían excesivamente extensas, con suplementos que solo les interesarían a determinados miembros de la red.

La variante de contrato interparte con cláusulas específicas para la red puede mantener la relación jurídica inicial con un grado de confidencialidad aceptable, pues solo se incluiría el nuevo contenido derivado de las negociaciones colectivas a nivel de red, por lo que esta se estima como la más recomendable dado el equilibrio que representa. Su puesta en práctica implicaría un período previo de negociaciones entre los miembros de la red de frutales y granos, a fin de extraer los criterios comunes a todos, los cuales formarán parte de los contratos que cada parte suscriba con la otra, y el resto del acuerdo se mantendría solo entre las piezas del eslabón.

Sería importante que cada integrante de la red tuviera los datos de localización del resto, aunque fuera de manera digital para ahorrar recursos.
Igualmente, debe quedar abierta la posibilidad de negociar los criterios comunes a la red ante cambios relevantes, tales como la afectación por plagas o eventos meteorológicos.

A continuación, se propone un ejemplo de instrumento que permite medir el grado de satisfacción de clientes, proveedores y operadores del Derecho, en cuanto al procedimiento de perfeccionamiento de la red desde el contrato.

El Grupo X desea conocer su grado de satisfacción con el procedimiento para perfeccionar la contratación en redes de producción y/o servicios, por lo cual se le ruega responder las siguientes preguntas de manera anónima. Le agradecemos de antemano por su colaboración.

1. ¿Ha notado usted cambios formales y prácticos en la contratación en redes de producción y/o servicios? ¿Cuáles?

2. ¿A partir de qué momento notó los cambios?

3. ¿El procedimiento aplicado le acarreó alguna dificultad? Descríbala por favor.

4. En una escala del 1 al 10, ¿cómo valora su nivel de satisfacción respecto al procedimiento?

5. ¿Tiene alguna otra sugerencia para perfeccionar el procedimiento?

\section{CONCLUSIONES}

Los contratos presentes en las redes agrarias están desarrollándose en un ambiente con limitaciones hacia lo interno y lo externo. A pesar de los recientes cambios en las relaciones de Cuba con el resto del mundo, el bloqueo persiste. La crisis económica mundial, las guerras y el cambio climático, entre otros factores, han ocasionado el encarecimiento de los precios de los alimentos e insumos a entregar a los productores, los cuales dificultan su despegue definitivo. Es por ello que urge trabajar con fuerza en los factores internos que pueden resolver los miembros de la red, comenzando por el perfeccionamiento de la comunicación interna, que hoy es deficiente, y el fomento de la creatividad o inventiva ante la necesidad de alternativas que suplan la carencia de materias primas o implementos de labranza.

Los juristas que elaboran y controlan los contratos, a pesar de los cambios implementados a partir de los lineamientos, necesitan desprenderse del formalismo y mecanicismo que impera en su actuar, pero también necesitan mejorar sus condiciones de trabajo. En este momento, existe un círculo vicioso 
en torno a este fenómeno difícil, pero no imposible de romper. Los implementos que aportan las entidades a las que pertenecen los consultores, asesores y abogados de bufete son siempre insuficientes. Dicha situación puede aliviarse con una mayor colaboración de los miembros de la red, quienes son los que tienen los recursos materiales a su alcance para facilitarles transportación, alimentación o materiales de oficina a los operadores del Derecho que los atienden, quienes a veces emplean todo un día en llegar y regresar de los centros de producción y/o distribución.

Se determinó que el procedimiento para diagnosticar la red agraria fue integrado con éxito a las otras propuestas que desarrollaron economistas, ingenieros industriales, contadores, informáticos y demás especialistas que participaron en su implementación. También se estableció que fue terminado el diagnóstico con éxito en las provincias de Santiago de Cuba y Sancti Spíritus. Dado su éxito, se está proponiendo su validación en el 2015, en La Habana, como parte de un Proyecto Nacional de Desarrollo Local. Por tanto, las herramientas ofrecidas como parte del procedimiento hacen que el contrato refleje en mayor medida la realidad de los actores involucrados y sus necesidades.

Las medidas de flexibilización del sistema económico son realmente revolucionarias cuando se mira al pasado: sin embargo, romper con más de cincuenta años de pensamiento tomará mucho tiempo. Los autores de este artículo coinciden en que los obstáculos en las redes agrarias, en general, tienen una raíz histórica, razón por la cual su desarrollo exitoso requerirá de paciencia y tesón, con el fin de no resignarse a dejar los problemas anquilosados.

\section{REFERENCIAS}

Decreto 283 de 2009. Reglamento de la Ley de Seguridad Social. Abril 24 de 2009. GoE N. ${ }^{\circ} 013$.

Decreto Ley 305 de 2012. De las Cooperativas no Agropecuarias. Diciembre 11 de 2012. GOE N. ${ }^{\circ} 53$.

Decreto Ley 306 de 2012. Del régimen especial de seguridad social de los socios de las Cooperativas no Agropecuarias. Diciembre 11 de 2012. GOE N. ${ }^{\circ} 53$.
Decreto 309 de 2012. Reglamento de las Cooperativas no Agropecuarias de primer grado. Diciembre 11 de 2012. GOE N. ${ }^{\circ} 53$.

Dirección de Asesoramiento Jurídico del Ministerio de Justicia. Situación ACTUAL DE LA ACTIVIDAd DE ASESORAMIENTO JURÍDICO EN LAS ENTIDADES QUE INTEGRAN LA ECONOMía nacional. Ministerio de Justicia. (2012).

Dunia Castillo Galán. Obsolescencia programada. JuvenTUd REBELDE, EDICIÓN Digital. Mayo 15 de 2013.

Silvia García Méndez, Mercedes de Armas Alonso y Karima Sánchez Rey. Miradas CRUZAdas: COMUNiCACIÓN Y cultura jurídica. Anuario del Centro de InvesTIGACIONES JURÍDICAS 2009-2010. Centro de Investigaciones Jurídicas. (2010).

Gerardo Machado, et al. El ASESORAMIENTO JURídico a LAS COOPERATIVAS AGRARIAS. INFORME FINAL DE INVESTIGACIÓN. Centro de Investigaciones Jurídicas. (2013).

Kathleen Fitzpatrick. Planned Obsolescence: PubliShing, Technology, AND the fUture of the AcaDEMY. New York University Press. (2011).

Ley 105 de 2009. Ley de Seguridad Social. Enero 22 de 2009. GOE N. ${ }^{\circ} 004$.

Ministerio de la Agricultura. Políticas y procedimientos A SEGUIR PARA LA CONTRATACIÓN DE LA PRODUCCIÓN AGROPECUARIA Y FORESTAL PARA EL AÑO 2014. Ministerio de la Agricultura. (2013)

Óscar Arambulo Reyes. Ensayo de la obsolescencia programada. Instituto de Postgrado y Educación Continua, Universidad de Guayaquil. (2013).

Partido Comunista de Cuba. Lineamientos de LA PolítiCa económica y social del Partido y la Revolución. Partido Comunista de Cuba. (2011).

Resolución 570 de 2012. Para licitar los bienes de un establecimiento estatal para su gestión por las Cooperativas no Agropecuarias. Diciembre 11 de 2012. GOE N. 53.

Resolución 427 de 2012. Que estableció indicaciones para el régimen tributario de los cooperativistas no agropecuarios. Diciembre 11 de 2012. GOE N. ${ }^{\circ} 53$.

Rodolfo Dávalos Fernández. ¿Embargo o bloqueo? LA INSTRUMENTACIÓN DE UN CRIMEN CONTRA CUBA. Capitán San Luis. (2012). 